\title{
A Feedback Strategy for Binary Symmetric Channels ${ }^{1}$
}

\author{
Aslan Tchamkerten, Emre Telatar \\ LTHI-ISC-I\&C-EPFL \\ CH-1015 Lausanne, Switzerland \\ e-mail: \{Aslan.Tchamkerten, Emre.Telatar\}@epfl.ch
}

\begin{abstract}
Communication over unknown Binary Symmetric Channels with instantaneous and perfect feedback is considered. We describe a universal scheme based on a decision feedback strategy.
\end{abstract}

\section{INTRODUCTION}

By means of variable length channel codes, a transmitter and a receiver communicate over a BSC with unknown crossover probability $\varepsilon$ with instantaneous and perfect feedback. At each time the transmitter sends a symbol according to the message he wants to send and the feedback it has received until that time. Based on a common stopping rule involving only the received sequence, the receiver and the transmitter decide when to decode. Since the decoding time is not fixed in advance, the rate of communication is a random variable which may depend on the channel. We wish to have a system whose long term rate comes close to the capacity of the channel over which it is used. The case of non-universal schemes is considered in [1]; in [2] feedback schemes are studied in general, here we describe a particularly simple scheme.

One possible strategy is to use a training sequence to measure the channel parameter and then to use a code designed for that parameter. However, the error probability of such a strategy is controlled by the length of the training sequence. Since this length needs to be negligible compared to the length of the information carrying sequence so that no rate is lost, the error exponent of such a scheme will be poor. Here we consider a strategy that does not use training sequences.

\section{EnCoding ANd Decoding SCheme}

Consider $M$ possible messages. The codebook is obtained by generating $M$ infinite sequences $\left\{\left\{x_{i}(m)\right\}_{i \geq 1}\right\}_{m=1}^{M}$ with the $x_{i}$ 's i.i.d. satisfying $\operatorname{Pr}\left(x_{i}=1\right)=\operatorname{Pr}\left(x_{i}=0\right)=\frac{1}{2}$. To communicate message $m$, the transmitter successively sends the bits of the sequence $x(m)$ until the receiver decides to decode. At time $n$, having received the sequence $y_{1}^{n}=y_{1}, \ldots, y_{n}$, the receiver computes $M$ sequences $\left\{\left\{z_{i}(m)\right\}_{i=1}^{n}\right\}_{m=1}^{M}$ according to $z_{i}(m)=x_{i}(m) \oplus y_{i}$. Clearly, for the true message $m$, $z_{i}(m)$ is a $\operatorname{Bernoulli}(\varepsilon)$ sequence, while for $m^{\prime} \neq m, z_{i}\left(m^{\prime}\right)$ is Bernoulli $\left(\frac{1}{2}\right)$. It follows that in order to correctly decode at time $n$, the receiver has to recognize which among the $M$ sequences $\left\{\left\{z_{i}(m)\right\}_{i=1}^{n}\right\}_{m=1}^{M}$ is not generated according to the Bernoulli $\left(\frac{1}{2}\right)$ distribution without knowing what $\varepsilon$ is.

Here we propose a stopping rule defined by a time-varying threshold. At each time $n$, the receiver computes the empirical frequencies of the 1's for each of the $M$ sequences $\left\{\left\{z_{i}(m)\right\}_{i=1}^{n}\right\}_{m=1}^{M}$. Decision is made as soon as any one of these empirical frequencies falls below the threshold. This threshold is intimately related to the capacity expression of the $\mathrm{BSC}$, and is discussed next.

${ }^{1}$ This work was supported in part by the Swiss National Science Foundation in the framework of the National Competence Center in Research for Mobile Information and Communication Systems.
For a BSC with parameter $\gamma<\frac{1}{2}$ and capacity $C(\gamma)=$ $\ln 2-H(\gamma)$ the expected codeword length allowing vanishing error probability is lower bounded by $\frac{\ln M}{C(\gamma)}$. Let us define for any $0 \leq \gamma<\frac{1}{2}, \alpha>1$ and any integer $M \geq 1, n(\alpha, M, \gamma)=$ $\left\lceil\frac{\alpha \ln M}{C(\gamma)}\right\rceil$. Inverting this last function for a fixed $\alpha$ and $M$, define $\delta(\alpha, M, t)=\sup \left\{\gamma \in\left[0, \frac{1}{2}\right): n(\alpha, M, \gamma)=t\right\}$. It follows that for any $M$ and $\alpha$, the function $\delta(\alpha, M, \cdot)$ is defined on $\mathbb{N} \cap\left[\left\lceil\frac{\alpha \ln M}{\ln 2}\right\rceil, \infty\right)$, has range in $[0,1 / 2)$, is strictly increasing with $\lim _{t \rightarrow \infty} \delta(\alpha, M, t)=\frac{1}{2}$. The time-varying threshold is precisely this $\delta(\alpha, M, \cdot)$.

Assuming we have a BSC with $\varepsilon \neq \frac{1}{2}$, instantaneous and perfect feedback, we have the following decoding algorithm:

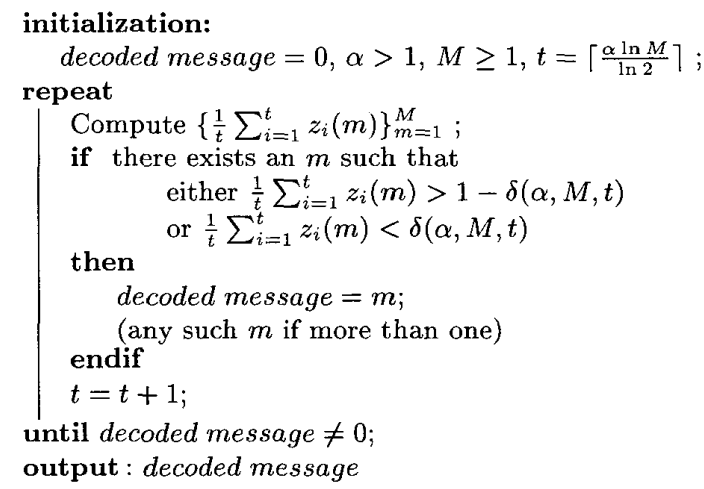

Note that as $\alpha$ is increased, the stopping criteria becomes harder to satisfy, and thus the rate of the code and the probability of error are decreased. Thus, $\alpha$ is the parameter that controls the tradeoff between reliability and rate. The performance of this encoding and decoding scheme is given in the following theorem:

Theorem. By using the above algorithm as the decoding rule for a BSC channel with parameter $\varepsilon \neq \frac{1}{2}$, any rate below capacity is achievable. Furthermore, the average probability of error of the ensemble of codes satisfies

$$
\operatorname{Pr}(\text { error }) \leq e^{-n\left[C(\varepsilon)-R-O\left(\frac{\ln n}{n}\right)\right]}
$$

where $n$ is the average decoding time, $C(\varepsilon)$ the capacity of the effective channel and $R=n^{-1} \ln M$ is the long term rate of the code.

\section{ACKNOWLEDGMENTS}

The authors are grateful to Marat Burnashev for helpful discussions and comments.

\section{REFERENCES}

[1] M. V. Burnashev, "Data transmission over a discrete channel with feedback: Random transmission time," Problems of Information Transmission, vol. 12, number 4, pp. 250-265, 1976.

[2] J. M.-S. Ooi: "A Framework for Low-Complexity Communication over Channels with Feedback", Ph.D. Thesis, Massachusetts Institute of Technology, 1997. 